\title{
Minimally Invasive Spine Surgery
}

\author{
Darren R. Lebl ${ }^{1}$
}

Published online: 5 August 2017

(C) Springer Science+Business Media, LLC 2017

The concept of minimally invasive surgery has gained increasing popularity in the last several decades in large part due to the great leaps forward witnessed by many subspecialties. The triumphs of minimally invasive innovation have included the transition from the morbidity of median sternotomy, or thoracotomy for open cardiothoracic procedures, to percutaneous catheter-based cardiac vessel and valve procedures, and the transition from open arthrotomy during sport procedures in the shoulder or knee to a specialty dominated almost entirely by the arthroscope. However, many fields of surgery have seen a less dramatic, more gradual transition. Spinal surgery has trended for years towards smaller incisions and biologic materials that minimize surgical duration and approachrelated morbidity. However, cases of complex spinal anatomy and deformity in particular have yet to broadly embrace a minimally invasive evolution.

The spine surgical community is often reminded that unless the surgeon's ability to perform a minimally invasive procedure can be done safely and effectively, satisfactory patient outcomes may be jeopardized. Complications associated with minimally invasive spine surgery such as violation of the cranial (non-fused) facet joint during placement of percutaneous pedicle screws, non-union related to inadequate discectomy and bone grafting during minimally invasive transforaminal lumbar interbody fusion, and nerve injury related to inadequate visualization during decompression may often be avoided simply by improving visualization through a larger surgical approach. While minimally invasive spine surgery

Darren R. Lebl

LeblD@hss.edu

1 Spine and Scoliosis Surgery, Hospital for Special Surgery, 535 East $70^{\text {th }}$ Street, New York, NY 10021, USA holds promise for lower blood loss, faster patient recovery, shorter hospital stays, and the potential to transition procedures to the ambulatory setting, safety in spinal surgery remains paramount and has (appropriately) tempered some of the enthusiasm for the results of aggressive early adapters.

Nonetheless, as the baby boomers (those born between 1946 and 1964) reach retirement age, the elderly population will continue to explode with an estimated one in five Americans in the "elderly" category by 2030. This demographic shift and the associated ballooning entitlement commitment will provide additional impetus for cost-effective spine surgery which will certainly be aided by minimally invasive techniques. Whether it is through specialized dilators and retractor systems or micro-endoscopic visualization, the concepts of preservation of the soft tissues, utilization of the body's natural tissue planes, avoidance of violation or surgical reflection of muscle tissue will remain important principles in the continued advancement of spine surgery.

The rapid proliferation in recent years of lumbar interbody fusion with specialized retractors through lateral or "extreme" lateral techniques provides a recent example of success of the minimally invasive philosophy in spine surgery. The ability to surgically access multiple lumbar (and thoracolumbar) segments of the spine for fusion through a few-centimeter incision and a relatively bloodless soft tissue plane was for all intents and purposes unachievable prior to the advent of these techniques. Additionally, the improved ability to provide an "indirect" foraminal decompression through restoring disk space height and neuroforaminal geometry, and placement of more anatomic cages to reconstruct the disk space has even the most obstinate of open surgery or "posterior-only" spine surgeons taking notice.

The major driver of cost in musculoskeletal care is without question the acute inpatient hospitalization. A transition towards outpatient surgery holds incentives not only for doctors 
and patients but also for payers. Currently, microdiscectomy, one- and two-level and anterior cervical discectomy and fusion (ACDF), and laminectomy are being commonly performed in an ambulatory setting. In so doing, many have reported a significantly lower cost for the entire episode of care. In select patients, one- and two-level lumbar fusions are even being performed in the ambulatory setting. For the time being, many elderly patients and/or those with certain medical comorbidities will remain more appropriate candidates for inpatient surgery such that appropriate perioperative medical care may be provided. However, as a general rule, the more spine surgery is transitioned out of the inpatient setting, the lower the cost to the health-care system, a fact that may be the most significant thrust behind the growth of minimally invasive techniques.

Advances in material science and a growing acceptance of the benefits of biologics in select patients are lowering the need for donor site morbidity from autograft harvest. Large structural autografts has largely been abandoned in favor of synthetic materials and biologics. For instance, 3D printed cages are already finding their way into the operating room with customized designs for individual patient anatomy and a porous architecture with the purported advantage of improving boney osseointegration. Recent in vitro studies have raised concern about the osseointegrative properties of polyetheretherketone (PEEK). These data suggest that the hydrophobicity of PEEK may serve as a suboptimal interface with the host bone leading to fibrous encapsulation and decreased fusion rates. This has precipitated a surge in the popularity of titanium implants as a substitute. However, conventional titanium implants are not without limitation. Significant artifacts exist that may interfere with postoperative imaging at the surgical site. Advancements in material science have yielded more porous types of titanium with less imaging artifacts and favorable osseointegration properties, and more recently, porous PEEK is under study.

Sophisticated software, improved sensors, and more facile intraoperative imaging have produced undeniable market growth and worldwide expansion of the image-guided navigation and robotic surgical markets. Robots have been integrated into the operating room for several decades now and have gained widespread acceptance and implementation in fields such as urologic and general surgery. Technological advances allow robotically assisted minimally invasive surgery in which the surgeon either controls the robotic instruments through a direct telemanipulator or through computerized control. A telemanipulator is a remote interface that allows the surgeon to perform surgical maneuvers while the robotic arms use end effectors to perform the actual tissue manipulation and procedure. In contrast, computercontrolled systems allow the surgeon to use a computer to control the robotic arms which don't require the surgeon to be physically present which opens up the possibility of remote surgery. Additionally, robotic technologies have the potential to enhance open surgical techniques with "smart" instruments and retractors that may reduce or eliminate tissue trauma through sensors that provide feedback control data. However, these technologies remain in their infancy and have yet to reach the tipping point of widespread acceptance for spine surgical indications. Cost remains a significant impediment to adoption in many centers, however, increased competition in the robotic segment will eventually drive down cost thereby making utilization of these technologies more commonplace. An exciting prospect to say the least.

The growing body of literature supporting the importance of spinal alignment in health-related quality of life measures following spinal surgery has led to a number of implant manufacturers developing platforms that allow spinal rods to be "customized" to each individual patient through software either pre- or intra-operatively. This already permits the "dialing-in" of more precise sagittal or coronal alignment and the potential to introduce these rods through smaller surgical incisions. Coupled with imaging, robotic, and material science advances, continued innovation is likely to increase adoption of these technologies in less invasive procedures.

While the mass marketing of minimally invasive spine surgery may seem a relatively new phenomenon, the concept of the minimization of collateral morbidity through the surgeon's own efforts is one that dates back to the times of the first physicians (primum non nocere-first, do no harm). Indeed, the future of minimally invasive spine surgery continues to be bright. The coming technological and procedural advances will certainly include the minimally invasive philosophy. The future will inevitably continue to incorporate less invasive techniques, robots, "smart instruments," imaged-guided navigation, biologics, and computers into the operating room. However, it is the surgeon that must shepherd in this new era with patient safety and clinical outcomes at the forefront. 